\title{
Endothelial restoration of CAD GWAS gene PLPP3 by nanomedicine suppresses YAP/TAZ activity and reduces atherosclerosis in vivo
}

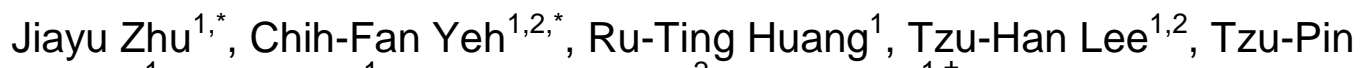

Shentu ${ }^{1}$, David Wu ${ }^{1}$, Kai-Chien Yang ${ }^{2,}$, Yun Fang ${ }^{1, \dagger}$

1. Department of Medicine, Biological Sciences Division, The University of Chicago

2. Department of Internal Medicine, National Taiwan University Hospital

*Equal contribution

${ }^{\dagger}$ Corresponding author: Yun Fang

Email: yfang1@medicine.bsd.uchicago.edu

ORCID: 0000-0003-4597-3095 (Yun Fang)

\section{Author Contributions}

J.Z., C.F.Y., R.T.H., T.H.L., T.P.S., and D.W. planned and executed experiments, analyzed data and interpreted results. K.C.Y. and Y.F. planned experiments and interpreted results. J.Z., C.F.Y., R.T.H., T.P.S., D.W, K.C.Y., and Y.F wrote and edited the manuscript.

\section{Competing Interest Statement:}

There authors declare no competing interests.

\begin{abstract}
Genome-wide association studies (GWAS) have suggested new molecular mechanisms in vascular cells driving atherosclerotic diseases such as coronary artery disease (CAD) and ischemic stroke (IS). Nevertheless, a major challenge to develop new therapeutic approaches is to spatiotemporally manipulate these GWAS-identified genes in specific vascular tissues in vivo. YAP (Yes-associated protein) and TAZ (transcriptional coactivator with PDZbinding motif) have merged as critical transcriptional regulators in cells responding to biomechanical stimuli, such as in athero-susceptible endothelial cells activated by disturbed flow (DF). The molecular mechanisms by which DF activates while unidirectional flow (UF) inactivates YAP/TAZ remain incompletely understood. Recent studies demonstrated that DF and genetic predisposition (risk allele) of CAD/IS locus 1 p32.2 converge to reduce phospholipid phosphatase 3 ( $P L P P 3$ ) expression in vascular endothelium. Restoration of endothelial PLPP3 in vivo, although remains challenging and unexplored, is hypothesized to reduce atherosclerosis. We devised a nanomedicine system integrating nanoparticles and $C d h 5$ promoter-driven plasmids to successfully restore PLPP3 expression in activated endothelium, resulting in suppressed
\end{abstract}


YAP/TAZ activity and reduced DF-induced atherosclerosis in mice. Mechanistically, our studies discovered a molecular paradigm by which CAD/IS GWAS gene PLPP3 inactivates YAP/TAZ by reducing lysophosphatidic acid (LPA)-induced myosin II and ROCK in endothelium under UF. These results highlight a new mechanistic link between GWAS and YAP/TAZ mechanoregulation and moreover, establish a proof of concept of vascular wall-based therapies employing targeted nanomedicine to manipulate CAD/IS GWAS genes in vivo.

\section{Main Text}

Genome-wide association studies (GWAS) and subsequent mechanistic investigations have identified novel cell-specific molecular mechanisms causing atherosclerosis. A major challenge for GWAS-informed, new anti-atherosclerotic therapeutics is to spatiotemporally manipulate GWAS genes in specific tissues of interest in vivo. GWAS identified chromosome 1 p32.2 as one of the most strongly associated loci with atherosclerotic diseases such as coronary artery disease (CAD) and ischemic stroke (IS). Our recent results $(1,2)$ highlighted phospholipid phosphatase 3 (PLPP3), located in 1p32.2, in endothelial mechano-transduction mechanisms associated with the focal nature of atherosclerosis in arterial curvatures and branches. While athero-protective unidirectional flow (UF) increases endothelial PLPP3, disturbed flow (DF) and CAD/IS risk allele at 1 p32.2 converge to suppress PLPP3 in endothelium prone to atherosclerosis. Endothelial PLPP3 restoration in vivo, although remains challenging, is expected to lessen DF-induced atherosclerosis.

Yorkie homologues YAP (Yes-associated protein) and TAZ (transcriptional coactivator with PDZ-binding motif) recently emerged as critical transcriptional regulators in cellular mechano-transduction such as DF-induced endothelial activation at atherosclerosis-prone sites $(3,4)$. Regulatory mechanisms linking human genetics to flow-dependent YAP/TAZ activity have not been proposed. We first discovered that the CAD/IS GWAS gene PLPP3 is a novel negative regulator of YAP/TAZ. PLPP3 inactivates YAP/TAZ in vascular endothelium under UF. Suppression of UF-induced PLPP3 using siRNA significantly increased YAP/TAZ activity, demonstrated by increased mRNA of YAP/TAZ downstream targets CTGF and CYR61 (Fig. 1A) and increased YAP dephosphorylation and CYR61 protein (Fig. 1B). We hypothesized that YAP/TAZ inactivation by PLPP3 is due to its phosphatase activity to hydrolyze lysophosphatidic acid (LPA). Indeed, treatments with Brp-LPA, a pan-LPA receptor antagonist and an inhibitor of Autotaxin, significantly abrogated the YAP dephosphorylation in PLPP3 knock-downed HAEC (Fig. 1C). Inhibition of myosin II and ROCK by Blebbistatin and Y27632 respectively, increased phospho-YAP in PLPP3 knock-downed HAEC (Fig. 1C). These data demonstrated for the first time, UF-induced PLPP3 inactivates endothelial YAP/TAZ by reducing LPAinduced signaling and myosin II and ROCK activities. 
Our in vitro mechanistic investigations discovered an unrecognized molecular link between an atherosclerotic GWAS gene and YAP/TAZ at the interface of mechano-transduction. To establish a proof of principle to spatially manipulate PLPP3 and YAP/TAZ signaling in vascular endothelium and treat atherosclerosis in vivo, we devised a method integrating nano-size carriers and endothelium-specific PLPP3-expressing plasmids. Nanoparticles were formulated by complexing positively-charged polyethylenimine (PEI) and negatively-charged plasmids, in which PLPP3 was driven by a $\sim 2.2 \mathrm{~kb}$ endothelium-specific $C D H 5$ (VE-Cadherin) promoter. Nanoparticles encapsulating CDH5-PLPP3-expressing plasmids, but not the control plasmids, significantly increased PLPP3 in endothelium-enriched intima isolated from the partially carotid ligated (PCL)-artery without affecting PLPP3 in the media/adventitia, showing endothelium-specific overexpression (Fig. 1D). Endothelial nuclear YAP in the PCL-artery was reduced by CDH5-PLPP3expressing plasmids (Fig. 1D). Nanoparticle-mediated endothelial PLPP3 restoration in the PCL-artery significantly attenuated DF-induced CTGF and CYR61 expression, and reduced atherosclerosis in Apoe ${ }^{-/}$mice (Fig. 1E).

Emerging results demonstrate that many GWAS genes have tissuespecific effects, underscoring the importance to integrate newly discovered GWAS mechanisms with targeted drug delivery systems. Here we demonstrate a proof of concept to spatially manipulate the atherosclerotic GWAS gene PLPP3 in vascular endothelium where PLPP3 uniquely responds to hemodynamics. Moreover, our results discovered a novel mechanistic link between atherosclerotic GWAS and YAP/TAZ regulation at the interface of endothelial mechano-transduction. In vivo restoration of endothelial PLPP3 by targeted nanomedicine significantly inactivated YAP/TAZ signaling and reduced DFinduced atherosclerosis (Fig. 1F).

\section{References}

1. Wu C, et al. (2015) Mechanosensitive PPAP2B Regulates Endothelial Responses to Atherorelevant Hemodynamic Forces. Circ Res 117(4):e4153.

2. Krause MD, et al. (2018) Genetic variant at coronary artery disease and ischemic stroke locus $1 \mathrm{p} 32.2$ regulates endothelial responses to hemodynamics. Proc Natl Acad Sci U S A 115(48):E11349-E11358.

3. Wang $L$, et al. (2016) Integrin-YAP/TAZ-JNK cascade mediates atheroprotective effect of unidirectional shear flow. Nature.

4. Wang KC, et al. (2016) Flow-dependent YAP/TAZ activities regulate endothelial phenotypes and atherosclerosis. Proc Natl Acad Sci U S A 113(41):11525-11530. 

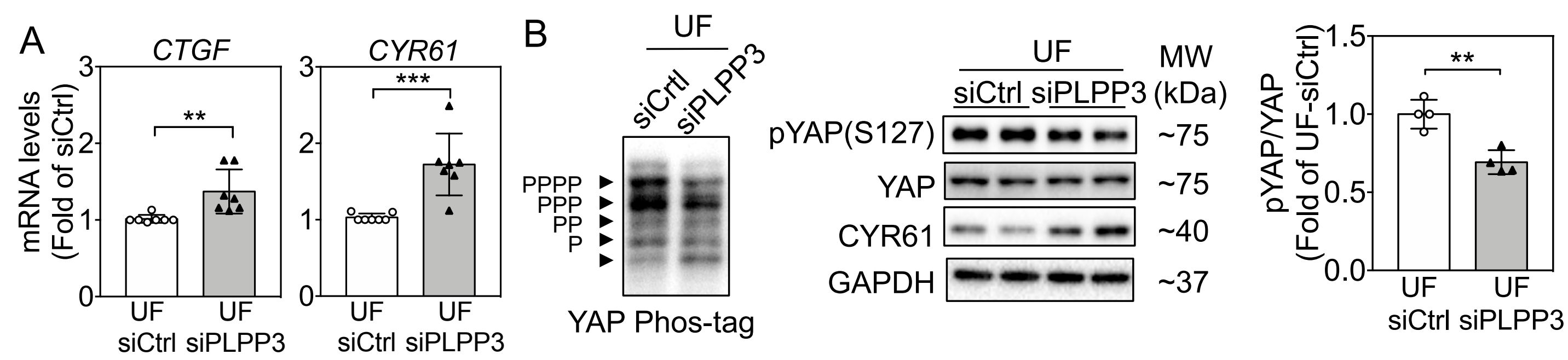

C

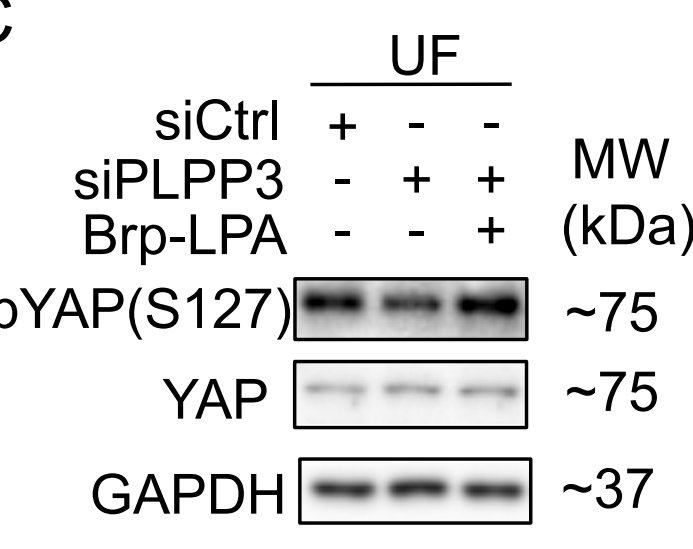

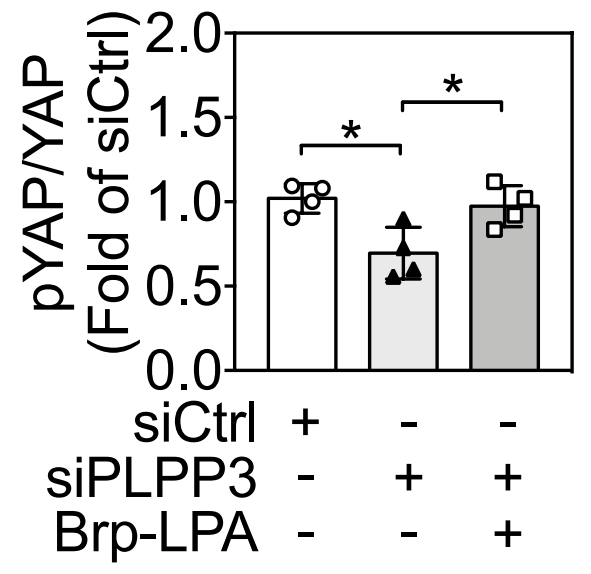

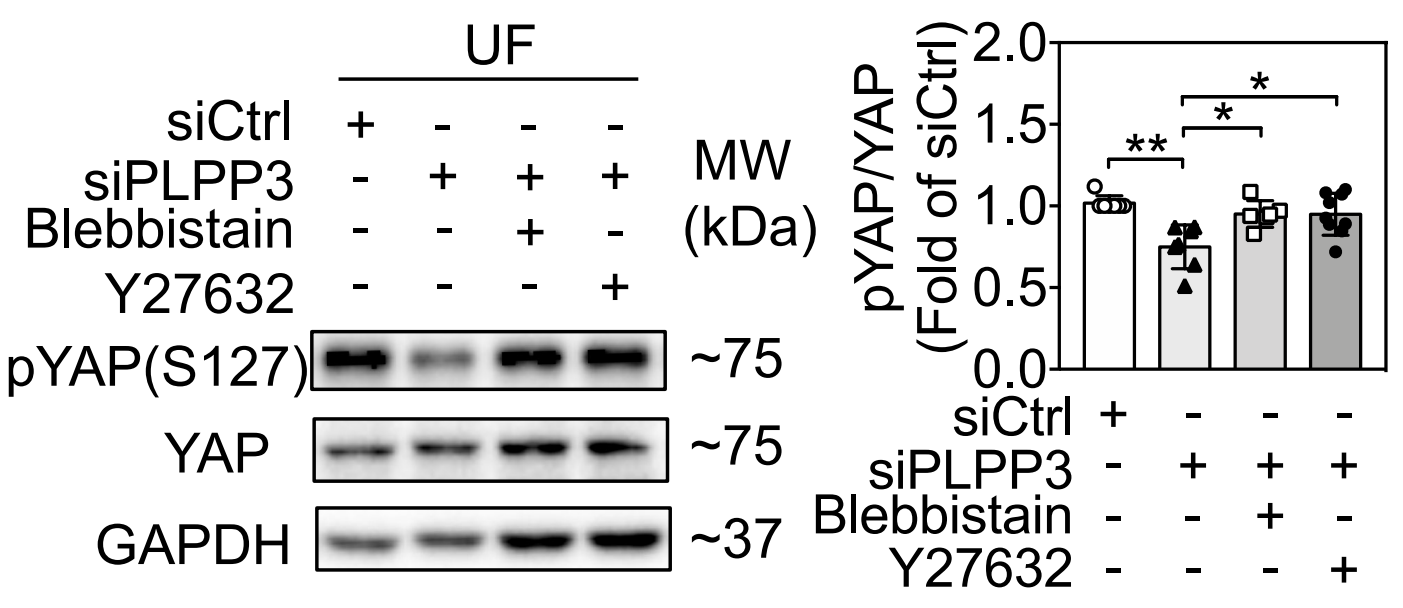


E

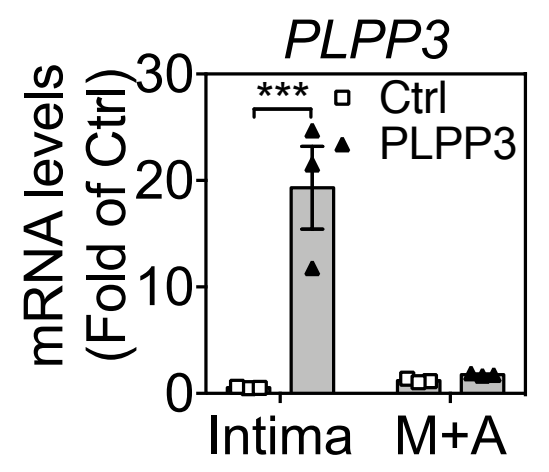

PEI nanoparticle

$$
\text { PLPP3 }
$$

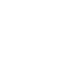

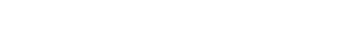

E

CDH5-driven plasmid i.v. injection

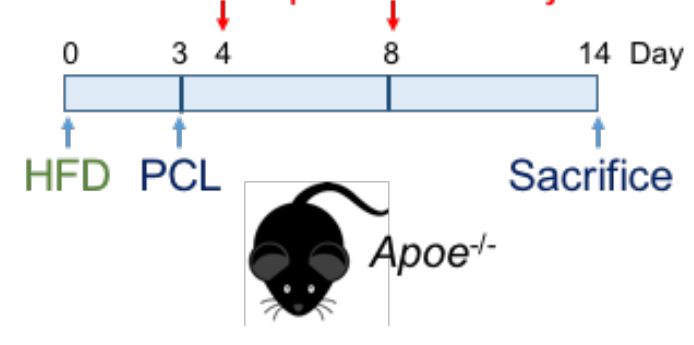

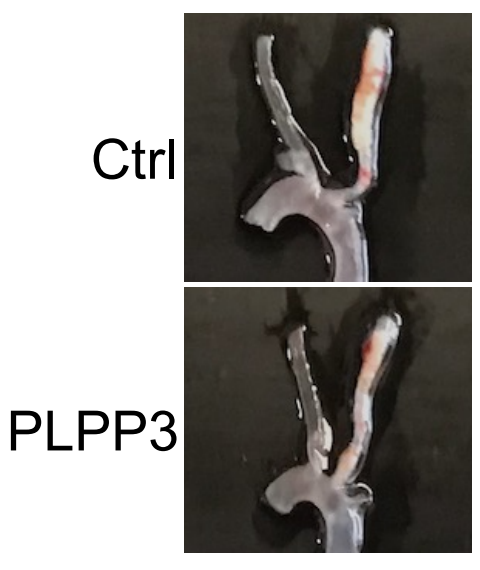

Ctrl

PLPP3

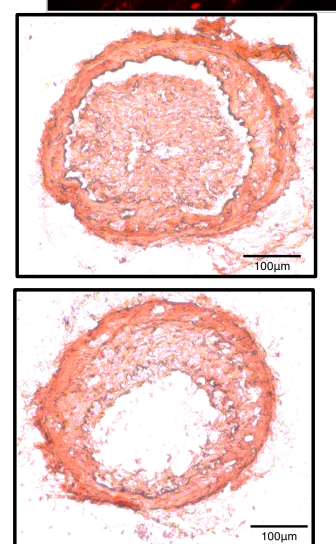

$\overline{10 \mu \mathrm{m}}$

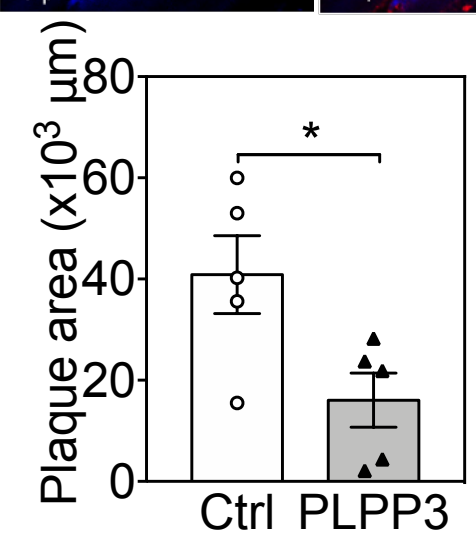

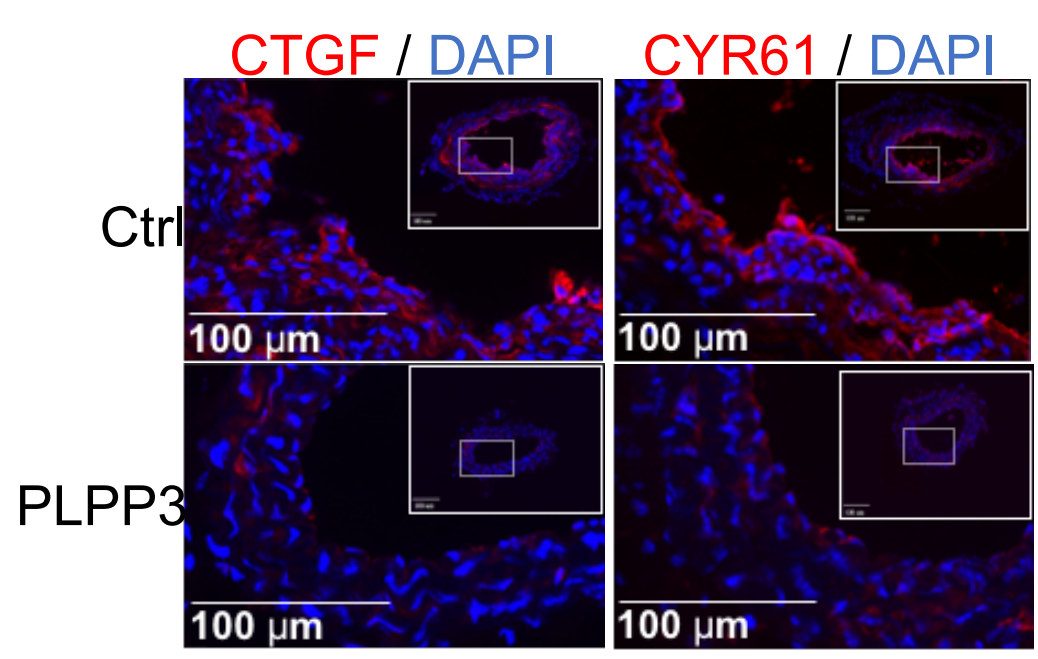
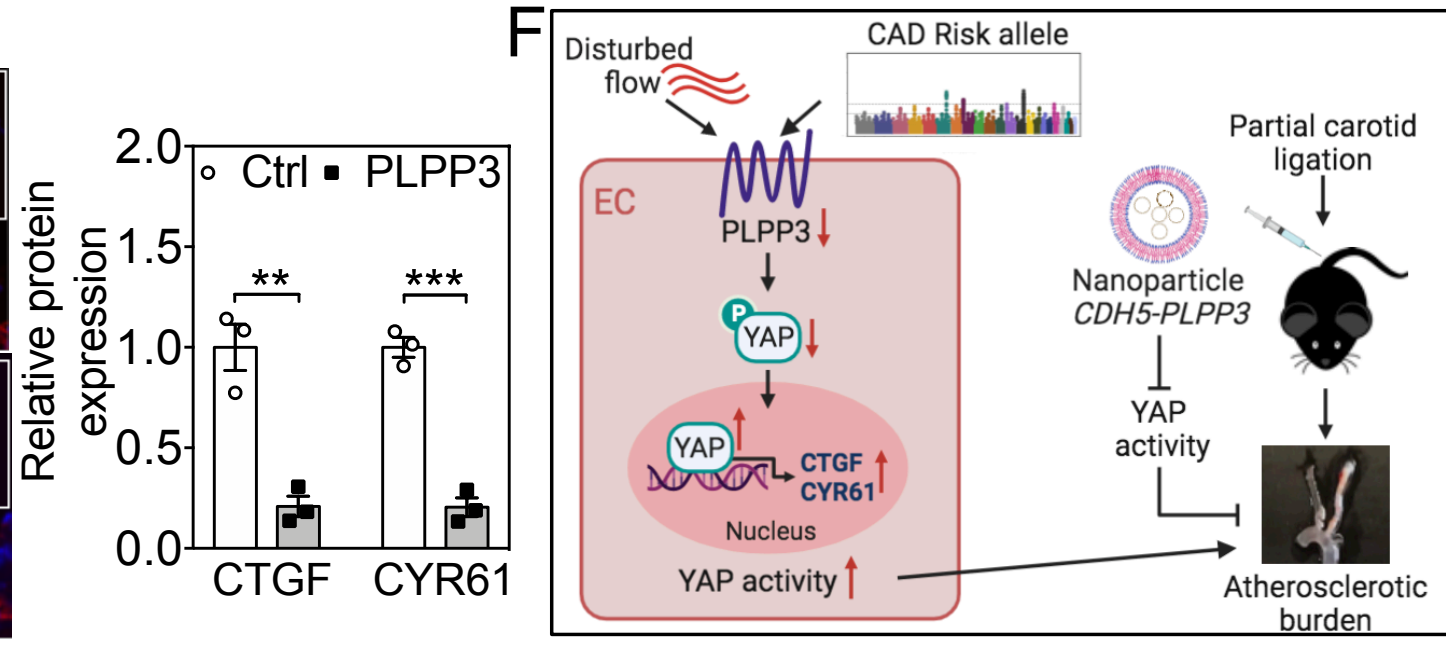
Figure 1. (A) Increased CTGF and CYR61 mRNA by PLPP3 knockdown in HAEC under UF ( $N=7$ ). (B) Decreased YAP phosphorylation, reduced PYAP/YAP ratio, and induced CYR61 protein by PLPP3 knockdown in HAEC under UF $(\mathrm{N}=4)$. (C) Increased pYAP/YAP ratio by Brp-LPA, ROCK inhibitor Y27632 (10 $\mu \mathrm{M})$ or myosin II inhibitor blebbistatin $(10 \mu \mathrm{M})$ in PLPP3 knock-downed HAEC under UF (N=4-9). (D) Increased PLPP3 mRNA in intima but not in media (M) and adventitia $(A)(N=3)$ in the PCL-artery in mice injected with nanoparticles containing CDH5-PLPP3 plasmids. Reduced endothelial nuclear YAP by PLPP3 overexpression. (E) Significantly reduced atherosclerosis and endothelial expression of CTGF and CYR61 in the PCL-artery in Apoe ${ }^{-/}$mice treated with nanoparticles containing CDH5-PLPP3 plasmids compared to those with $C D H 5$ only (Ctrl) plasmids $(\mathrm{N}=3-5)$. Data in $1 \mathrm{~A}-\mathrm{C}$ represent mean $\pm \mathrm{SD}$ while mean \pm SEM in Fig. 1D-E. * $P<0.05,{ }^{* *} P<0.005,{ }^{* * *} P<0.0005$. For two groups, unpaired student t-test was used. One-way ANOVA was used for multiple groups. 